\title{
Genome-wide analysis of haplotype interaction for the data from the North American Rheumatoid Arthritis Consortium
}

\section{Jungsun Park ${ }^{1}$, Junghyun Namkung ${ }^{1}$, Mina Jhun ${ }^{1}$ and Taesung Park*1,2}

Addresses: ${ }^{1}$ Interdisciplinary Program in Bioinformatics, Seoul National University, San56-l, Shin Lim-Dong, Kwan Ak-Ku, Seoul 151-747, Republic of Korea and ${ }^{2}$ Department of Statistics, College of Natural Science, Seoul National University, San56l, Shin Lim-Dong, Kwan Ak-Ku, Seoul 151-747, Republic of Korea

E-mail: Jungsun Park - pj518@snu.ac.kr; Junghyun Namkung - jh.namkung@gmail.com; Mina Jhun - mhj617@snu.ac.kr; Taesung Park* - tspark@stats.snu.ac.kr

${ }^{*}$ Corresponding author

from Genetic Analysis Workshop 16

St Louis, MO, USA 17-20 September 2009

Published: 15 December 2009

BMC Proceedings 2009, 3(Suppl 7):S34 doi: 10.1186/1753-656I-3-S7-S34

This article is available from: http://www.biomedcentral.com/I753-656I/3/S7/S34

(c) 2009 Park et al; licensee BioMed Central Ltd.

This is an open access article distributed under the terms of the Creative Commons Attribution License (http://creativecommons.org/licenses/by/2.0), which permits unrestricted use, distribution, and reproduction in any medium, provided the original work is properly cited.

\begin{abstract}
Recent genome-wide association studies on several complex diseases have focused on individual single-nucleotide polymorphism (SNP) analysis; however, not many studies have reported interactions among genes perhaps because the gene-gene and gene-environment interaction analysis could be infeasible due to heavy computing requirements. In this study we propose a new strategy for exploring the interactions among haplotypes. The proposed method consists of two steps. Step I tests the single-SNP association of whole genome with multiple testing corrections and finds the haplotype blocks of the significant SNPs. Step 2 performs interaction analysis of haplotypes within blocks. Our proposed method is applied to the rheumatoid arthritis data for Genetic Analysis Workshop 16.
\end{abstract}

\section{Background}

Complex diseases such as rheumatoid arthritis (RA) are the results of a complex interplay of genetic and environmental factors. Previously, a linkage study of Jawaheer et al. [1] showed that regions from chromosome 1 interact with chromosome 6, resulting in synergistic effect on risk of RA. Simulation studies showed that interaction analysis can be more powerful to detect disease associated genes than analysis ignoring interactions even after correction for multiple testing $[2,3]$. Methods based on haplotypes were also shown to provide additional power for mapping disease genes when compared with the analysis of individual singlenucleotide polymorphisms (SNPs) [4,5]. Because a haplotype comprises multiple SNPs on the same inherited chromosomes, the haplotype-based approaches can also provide insight into factors influencing the dependency among genetic markers. Such insight may provide information essential for understanding human evolution and also for identifying cisinteractions between two or more causal variants [6].

Recently, genome-wide association studies (GWAS) on several complex diseases were successfully conducted 
and some promising results with putative disease-related genes were reported [7-9]. However, analyses from those studies only considered individual SNPs, and not many studies have reported interactions among genes in GWAS because the gene-gene and gene-environment interaction analysis could be infeasible due to heavy computing times. For example, the North American Rheumatoid Arthritis Consortium (NARAC) data for the genetic data analysis workshop (GAW16) contains 545,080 SNPgenotype fields from the Illumina $550 \mathrm{k}$ chip. Note that more than $10^{11}$ interaction models are possible for all pairs of SNPs in this data set, which requires enormous computing times.

In this study, we propose genome-wide analysis of haplotype interaction (GWAHI) to discover interactions between unlinked regions based on haplotypes. The proposed GWAHI consists of the following two steps: Step 1 tests the single-SNP association of whole genome with multiple testing corrections and finds the haplotype blocks of the significant SNPs, and Step 2 performs interaction analysis of haplotypes within blocks. Our proposed GWAHI is applied to the RA data for GAW16.

\section{Methods \\ Data}

In the GAW16 RA data, genotypes for 545,080 SNPs from total 2,062 individuals were provided. The samples consisted of 868 patients and 1,194 controls. We excluded outliers of population stratification test performed by Egenstrat [10] and samples showing sex matching error tested by PLINK [11]. We also excluded SNPs with minor allele frequency less than $5 \%$ or with the significance level of $10^{-6}$ for Hardy-Weinberg equilibrium test. After data cleaning, 491,345 SNPs and 2,048 samples were used in our analysis.

\section{Single-SNP association test}

We tested for additive and co-dominant genetic models using PLINK [11]. For the additive model we used logistic-genotypic option in PLINK, which provided a test based on logistic regression; for the co-dominant model we used model option in PLINK, which provided a $\chi^{2}$ test. Both tests were performed over the whole genome. For multiple-testing correction, we applied the Bonferoni correction. SNPs were selected if they showed significant association results with $p$-values less than $1.03 \times 10^{7}$.

\section{Haplotype block construction and association test}

We constructed haplotype blocks for each chromosome using Haploview [12] and Gabriel's block definition. Next, we identified haplotype blocks containing one or more SNPs chosen from the individual SNP test. We also included haplotype blocks that showed significant association ( $p$-value $<5.35 \times 10^{-7}$ ). The haplotype association test was also performed by PLINK [11]. We used hap-assoc option in PLINK, which generated haplotype-specific tests (1 df).

\section{Haplotype interaction}

Association tests using haplotype interaction were conducted using the method introduced by Becker et al. [3] and implemented in program FAMHAP. FAMHAP uses the expectation-maximization algorithm to obtain maximum-likelihood estimates of the haplotype frequencies at each of the unlinked regions for pooled case-control samples. Then, a contingency table was constructed that comprises rows referring to disease status and columns referring to multi-region haplotype configurations. For association tests, the usual chi-square tests and permutation tests were performed [3].

\section{Results}

Single-SNP and haplotype association analysis

SNP and haplotype association analysis identified 411 significant SNPs and 146 haplotype blocks, among which 255 SNPs and 133 haplotypes were from chromosome 6. The distribution of the significant SNPs over the chromosome is shown in Figure 1. Among 411 SNPs, 219 SNPs overlapped and were included in the identified haplotype blocks. In order to see how much influence the significant SNPs have on the significance of the haplotype blocks, we calculated the correlation coefficient between the $p$-values of overlapped SNPs and haplotype blocks. After the logtransformation of $p$-values, the correlation coefficients were $0.7295\left(p\right.$-value $\left.<1.0 \times 10^{-15}\right)$ for the additive model and $0.8036\left(p\right.$-value $<1.0 \times 10^{-15}$ ) for the codominant model. Thus, we conclude that the significant SNPs have much influence on the significance of the haplotype blocks. We also computed the correlation coefficients focusing only on chromosome 6 . For the 211 overlapped SNPs, we obtained very similar correlation coefficients. When we mapped the significant SNPs using dbSNP database [13], 238 unique genes or putative genes were identified. Many SNPs and blocks were located in the human leukocyte antigen (HLA) regions, as presented in Table 1 . Some of our results reconfirmed the previous findings on genetic factors attributable to RA risk reported in the OMIM database [14]. For example, PTPN22 on chromosome 1, TRAF1 on chromosome 9, and NFKBIL1, HLA-C, and HLA-G on chromosome 6 were also detected by our association analysis. Pathway mapping of the 238 identified genes showed that 51 genes were matched with 60 KEGG pathways (Additional File 1), among which 18 KEGG pathways were related to immune responses and signaling pathways. 


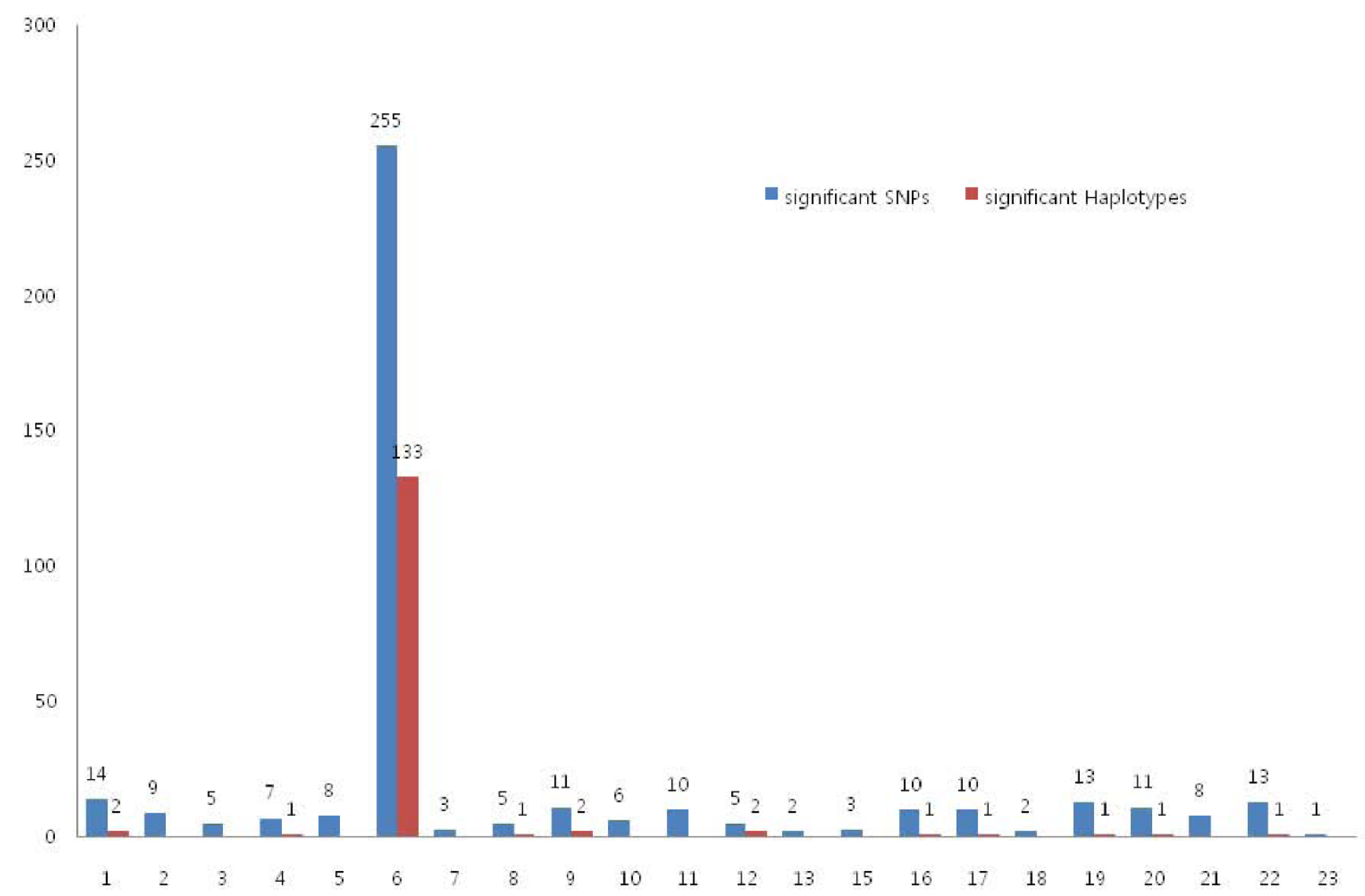

Figure I

The proportion of significant SNPs and haplotype blocks from the single association tests. In our single association analysis, $62 \%$ of the significant SNP and $91 \%$ of the significant haplotypes were located in chromosome 6 . There were no significant markers in chromosome 14.

Table I: The genes containing significant SNPs and haplotype blocks and comprising the HLA regions

\begin{tabular}{|c|c|c|c|}
\hline HLA class & Locus name & No. SNPs & No. haplotypes \\
\hline \multirow[t]{6}{*}{ Class I } & HLA-C & 1 & I \\
\hline & HLA-E & 0 & I \\
\hline & HLA-F & 0 & I \\
\hline & HLA-G & I & i \\
\hline & $H L A-H$ & 2 & 2 \\
\hline & HLA-80 & 1 & I \\
\hline \multirow[t]{11}{*}{ Class II } & HLA-DMA & 0 & I \\
\hline & HLA-DMB & 0 & I \\
\hline & HLA-DOA & 1 & 2 \\
\hline & HLA-DOB & I & 2 \\
\hline & HLA-DPAI & 4 & i \\
\hline & HLA-DQAI & 0 & i \\
\hline & HLA-DQA2 & I & I \\
\hline & HLA-DQBI & I & 0 \\
\hline & HLA-DQB2 & 0 & I \\
\hline & HLA-DRA & 0 & I \\
\hline & HLA-DRB9 & 2 & I \\
\hline
\end{tabular}

We identified the functional class of the significant SNPs, among which 23 SNPs were non-synonymous (Table 2). We then tested whether the amino-acid substitution caused by the non-synonymous SNPs affects protein function damaging using the SIFT program. SIFT predicts whether an amino acid substitution affects protein function based on sequence homology and the physical properties of amino acids [15].

We determined out that two non-synonymous SNPs were not functionally tolerant and were reported as linked to disease in OMIM [14]. One of the non-synonymous SNPs was rs2075800, which was reported to be related with sarcoidosis [16], an immune system disorder characterized by non-caseating granulomas [17]. The other SNP was rs2476601 in PTPN22, which was reported to be associated with four separate autoimmune phenotypes: type I diabetes mellitus, RA, systemic lupus erythematosus, and Hashimoto thyroiditis [18]. 
Table 2: The functional class of significant SNPs

\begin{tabular}{lc}
\hline Function class & No. SNPs \\
\hline Intron & 127 \\
UTR & 23 \\
Cds-nonsynonymous & 23 \\
Cds-synonymous & 7 \\
Locus & 32 \\
\hline
\end{tabular}

\section{Haplotype block construction and association test}

We started to construct haplotype blocks from the shortest chromosome 22 sequentially. Due to lack of memory space in our system (6 GB memory), computation was completed only for the chromosomes up to 18 . In order to handle memory problem, we split the whole chromosome into several short regions containing 10,000 SNPs (60 Mb). Each split region was defined to have 500 overlap SNPs (3 Mb) overlapping with its neighbor regions. Then we reconstructed the haplotype blocks for each region and merged them using the overlapping regions. Through the split-and-merge haplotype reconstruction, a total of 93,397 haplotype blocks were constructed. The average block length was 17055.5 base-pairs (median, 7638 base-pairs), and one block included 4.3 SNPs on average (median, 3 SNPs). Finally, 338 haplotype blocks were selected, which contained either significant SNPs or haplotype blocks.

\section{Haplotype interaction}

For Step 2, we conducted the interaction analysis for all pairs of the 338 haplotype blocks. The interaction analysis selected 46,995 haplotype pairs from 56,953 possible pairs (Bonferoni corrected $p$-value $<8.78 \times 10^{-7}$ ). Among the detected haplotype pairs, 13,563 were from chromosome $6 ; 25,300$ pairs included one haplotype from chromosome 6; 8,132 pairs were not from chromosome 6 . We then constructed a haplotype-based network graph in which each node represents a haplotype. This network graph illustrates an overview of haplotype interactions. Each edge represents an interaction of a pair of haplotypes. The more haplotypes connected, the more interactions exist between the haplotypes. We identified 39 hub haplotypes that highly interacted with other haplotypes. Twenty-two of the hub haplotype were located in 15 gene regions: PSORS1C1, BAT2, LY6G5C, BAT5, LY6G6D, TNXB, CREBL1, NOTCH4, LOC401252, C6orf10, BTNL2, HLA-DRA, HLA-DRB9, HLA$D Q A 1$, and TAP2. Most of the hub haplotypes were located in 6p21.33-6p21.32 (MHC III regions) except one from the chromosome 7 (rs11762043).

\section{Discussion}

RA is an inflammatory disease, primarily of the joints, with autoimmune features and a complex genetic component. Among the 411 SNPs we detected from the single-SNP analysis, only five were previously reported to be associated with RA. The other 406 SNPs are candidate SNPs associated with RA. We list the top ten SNPs in the Table 3; the remaining SNPs are summarized at our website [19]. The highlighted SNPs were selected by both the additive and codominant model. Our GWAHI analysis discovered interactions between haplotype blocks, especially 39 hub haplotypes included genes expected to be related to immune response and various signaling pathways. Further study of these genes may give us more insight into the mechanism of RA development.

Table 3: Significant SNPs from the SNP association tests

\begin{tabular}{|c|c|c|c|c|c|c|c|c|}
\hline dbSNP ID & Model & $p$-Value & Chr & Chr position & Allele & Strand & Gene symbol & Functional class \\
\hline rs2395 I 75 $5^{a}$ & codominant & $2.46 \times 10^{-112}$ & 6 & $32,5 \mid 3,004$ & $\mathrm{~A} / \mathrm{G}$ & + & & \\
\hline rs660895 & codominant & $5.05 \times 10^{-107}$ & 6 & $32,685,358$ & $\mathrm{~A} / \mathrm{G}$ & + & & \\
\hline rs691007I & codominant & $6.65 \times 10^{-94}$ & 6 & $32,390,832$ & $\mathrm{~A} / \mathrm{G}$ & + & C6orflo & intron \\
\hline rs2395 I 63 & codominant & $1.10 \times 10^{-90}$ & 6 & $32,495,787$ & $\mathrm{C} / \mathrm{T}$ & + & & \\
\hline rs37633 I 2 & codominant & $1.87 \times 10^{-86}$ & 6 & $32,484,326$ & $\mathrm{~A} / \mathrm{G}$ & + & BTNL2 & locus \\
\hline rs3763309 & codominant & $2.06 \times 10^{-86}$ & 6 & $32,483,951$ & $\mathrm{~A} / \mathrm{C}$ & + & BTNL2 & locus \\
\hline rs9275224 & codominant & $5.96 \times 10^{-86}$ & 6 & $32,767,856$ & $\mathrm{~A} / \mathrm{G}$ & + & & \\
\hline rs6457617 & codominant & $6.83 \times 10^{-75}$ & 6 & $32,771,829$ & $\mathrm{C} / \mathrm{T}$ & + & & \\
\hline rs927I568 & codominant & $1.44 \times 10^{-66}$ & 6 & $32,698,44 \mid$ & $\mathrm{A} / \mathrm{G}$ & + & & \\
\hline rs2395।85 & codominant & $8.68 \times 10^{-65}$ & 6 & $32,54|| 45$, & $\mathrm{G} / \mathrm{T}$ & + & & \\
\hline rs660895 & additive & $1.40 \times 10^{-62}$ & 6 & $32,685,358$ & $\mathrm{~A} / \mathrm{G}$ & + & & \\
\hline rs927I568 & additive & $5.33 \times 10^{-56}$ & 6 & $32,698,44 \mid$ & $\mathrm{A} / \mathrm{G}$ & + & & \\
\hline rs2395185 & additive & $3.30 \times 10^{-55}$ & 6 & $32,54 I, I 45$ & $\mathrm{G} / \mathrm{T}$ & + & & \\
\hline rs691007I & additive & $7.28 \times 10^{-55}$ & 6 & $32,390,832$ & $\mathrm{~A} / \mathrm{G}$ & + & C6orflo & intron \\
\hline rs2395 I 63 & additive & $2.58 \times 10^{-54}$ & 6 & $32,495,787$ & $\mathrm{C} / \mathrm{T}$ & + & & \\
\hline rs 2516049 & additive & $4.74 \times 10^{-53}$ & 6 & $32,678,378$ & $\mathrm{~A} / \mathrm{G}$ & - & & \\
\hline rs4775I5 & additive & $1.25 \times 10^{-52}$ & 6 & $32,677,669$ & $\mathrm{C} / \mathrm{T}$ & - & & \\
\hline rs3763309 & additive & $4.34 \times 10^{-52}$ & 6 & $32,483,951$ & $\mathrm{~A} / \mathrm{C}$ & + & BTNL2 & locus \\
\hline rs37633II & additive & $5.29 \times 10^{-51}$ & 6 & $32,484,326$ & $A / G$ & + & BTNL2 & locus \\
\hline rs2395 I 75 & additive & $6.53 \times 10^{-50}$ & 6 & $32,513,004$ & $\mathrm{~A} / \mathrm{G}$ & + & & \\
\hline
\end{tabular}

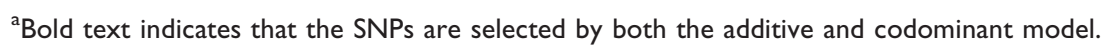


We provide a new strategy for exploring the interactions among haplotypes in genome-wide association analysis. Our proposed GWAHI narrowed down the set of important haplotype blocks for assessing evidence of interactions using a two-step analysis. For our haplotype interaction analysis, we included not only significant haplotype blocks but also haplotype blocks containing significantly associated SNPs. This could give us more insight of the relationships between the significant SNP and the significant halotypes.

We used the two-step approach to minimize the computational burden by reducing the number of haplotypes. In our analysis, the computational time was reasonable (12 hours using 10 nodes: Dual Core AMD Opteron(TM) Processor 270 CPU, 6 G memory). However, in this twostep approach the haplotype interactions without main effects cannot be detected at Step 1 and thus cannot be included in interaction analysis at Step 2. If we included these haplotypes in interaction analysis, then the computational time would increase exponentially due to the increase of the number of haplotypes. Thus, it is a challenge to handle this computational burden more efficiently for successful GAWHI.

Our haplotype interaction analysis was conducted using the program FAMHAP. The range of permutation $p$-values was dependent on the number of simulations. The $p$-value becomes zero when it is below $1.0 \times 10^{-4}$ in the default setting (simulation time, $1 \times 10^{4}$ ). Thus, the rank of the lowest $p$-values ( $p$-value $=0$ ) is unattainable. Even though we increased the simulation time to the $1.0 \times 10^{8}$, we still had the same problem. As the simulation time increased, the computing time increased exponentially (from 8 second for the default setting to 8 hours for the $1.01 .0 \times$ $10^{8}$ simulation time for one run). In order to get higher resolution of the corrected p-values, we computed the $p$-values of the chi-square statistics from FAMHAP program by using the chi-square distribution and then applied Bonferroni correction. In fact, the non-zero permutation $p$-values from FAMHAP did not differ much from the corrected $p$-values computed by chi-square statistics.

\section{List of abbreviations used}

GAW: Genetic Analysis Workshop; GWAHI: Genomewide analysis of haplotype interaction; GWAS: Genomewide association studies; HLA: Human leukocyte antigen; NARAC: North American Rheumatoid Arthritis Consortium; RA: Rheumatoid arthritis; SNP: Singlenucleotide polymorphism.

\section{Competing interests}

The authors declare that they have no competing interests.

\section{Authors' contributions}

JP carried out the data preprocessing and statistical analysis and drafted the manuscript. JN participated in the study design and helped to draft the manuscript. MJ participated in the data preprocessing. TP conceived of the study, participated in its design and coordination, and helped to draft the manuscript. All authors read and approved the final manuscript.

\section{Additional material}

\section{Additional file 1}

Genes matched to KEGG pathways among those identified by haplotype interaction analysis.

Click here for file

[http://www.biomedcentral.com/content/supplementary/17536561-3-S7-S34-S1.pdf]

\section{Acknowledgements}

This work was supported by the National Research Laboratory Program of Korea Science and Engineering Foundation (MI0500000I26) and fellowship of Seoul Citizen in Science.

This article has been published as part of BMC Proceedings Volume 3 Supplement 7, 2009: Genetic Analysis Workshop 16. The full contents of the supplement are available online at http://www.biomedcentral.com/ $\underline{1753-656 \mid / 3 \text { ? issue }=S 7 .}$.

\section{References}

I. Jawaheer D, Seldin MF, Amos Cl, Chen WV, Shigeta R, Etzel C, Damle A, Xiao X, Chen D, Lum RF, Monteiro J, Kern M, Criswell LA, Albani S, Nelson JL, Clegg DO, Pope R, Schroeder HW Jr, Bridges SL Jr, Pisetsky DS, Ward R, Kastner DL, Wilder RL, Pincus T, Callahan LF, Flemming D, Wener MH, Gregersen PK and North American Rheumatoid Arthritis Consortium: Screening the genome for rheumatoid arthritis susceptibility genes: a replication study and combined analysis of 512 multicase families. Arthritis Rheum 2003, 48:906-916.

2. Marchini J, Donnelly P and Cardon LR: Genome-wide strategies for detecting multiple loci that influence complex diseases. Nat Genet 2005, 37:4I3-4I7.

3. Becker T, Schumacher J, Cichon S, Baur MP and Knapp M: Haplotype interaction analysis of unlinked regions. Genet Epidemiol 2005, 29:313-322.

4. Akey J, Jin L and Xiong M: Haplotypes vs single marker linkage disequilibrium tests: what do we gain?. Eur J Hum Genet 200 I, 9:291-300.

5. Schaid DJ: Evaluating associations of haplotypes with traits. Genet Epidemiol 2004, 27:348-364.

6. Liu N, Zhang $\mathrm{K}$ and Zhao $\mathrm{H}$ : Haplotype-association analysis. Adv Genet 2008, 60:335-405.

7. Scott LJ, Mohlke KL, Bonnycastle LL, Willer CJ, Li Y, Duren WL, Erdos MR, Stringham HM, Chines PS, Jackson AU, ProkuninaOlsson L, Ding CJ, Swift AJ, Narisu N, Hu T, Pruim R, Xiao R, Li XY, Conneely KN, Riebow NL, Sprau AG, Tong M, White PP, Hetrick KN, Barnhart MW, Bark CW, Goldstein JL, Watkins L, Xiang F, Saramies J, Buchanan TA, Watanabe RM, Valle TT, Kinnunen L, Abecasis GR, Pugh EW, Doheny KF, Bergman RN, Tuomilehto J, Collins FS and Boehnke M: A genome-wide association study of type 2 diabetes in Finns detects multiple susceptibility variants. Science 2007, 3 I 6: I 34 I-1345.

8. Liu Y, Helms C, Liao W, Zaba LC, Duan S, Gardner J, Wise C, Miner A, Malloy MJ, Pullinger CR, Kane JP, Saccone S, Worthington J, Bruce I, Kwok PY, Menter A, Krueger J, Barton A, Saccone NL and Bowcock AM: A genome-wide association study of psoriasis and psoriatic arthritis identifies new disease loci. PLoS Genet 2008, 4:el00004I. 
9. The Wellcome Trust Case Control Consortium: Genome-wide association study of 14,000 cases of seven common diseases and 3,000 shared controls. Nature 447:66I-678.

10. Price AL, Patterson NJ, Plenge RM, Weinblatt ME, Shadick NA and Reich D: Principal components analysis corrects for stratification in genome-wide association studies. Nat Genet 2006, 38:904-909.

II. Purcell S, Neale B, Todd-Brown K, Thomas L, Ferreira MA, Bender D, Maller J, Sklar P, de Bakker PI, Daly MJ and Sham PC: PLINK: a tool set for whole-genome association and population-based linkage analyses. Am J Hum Genet 2007, 81:559-575

12. Barrett JC, Fry B, Maller J and Daly MJ: Haploview: analysis and visualization of LD and haplotype maps. Bioinformatics 2005, 2l:263-265.

13. dbSNP database. http://www.ncbi.nlm.nih.gov/projects/SNP/

14. OMIM: Online mendelian inheritance in man.http://www.ncbi. nlm.nih.gov/entrez/dispomim.cgi?id= I 80300 .

15. $\mathrm{Ng} \mathrm{PC}$ and Henikoff S: Accounting for human polymorphisms predicted to affect protein function. Genome Res 2002 , 1 2:436-446.

16. Spagnolo P, Sato H, Marshall SE, Antoniou KM, Ahmad T, Wells AU, Ahad MA, Lightman S, du Bois RM and Welsh KI: Association between heat shock protein 70/Hom genetic polymorphisms and uveitis in patients with sarcoidosis. Invest Ophthalmol Vis Sci 2007, 48:3019-3025.

17. Wikipedia: Sarcoidosis. http://en.wikipedia.org/wiki/Sarcoidosis.

18. Criswell LA, Pfeiffer KA, Lum RF, Gonzales B, Novitzke J, Kern M, Moser KL, Begovich AB, Carlton VE, Li W, Lee AT, Ortmann W, Behrens TW and Gregersen PK: Analysis of families in the Multiple Autoimmune Disease Genetics Consortium (MADGC) collection: the PTPN22 620W allele associates with multiple autoimmune phenotypes. Am J Hum Genet 2005, 76:56I-57I.

19. Significant SNP information. http://bibs.snu.ac.kr/ pi5/8/GAWI6/ SNPTable.html.

\section{Publish with Biomed Central and every scientist can read your work free of charge}

"BioMed Central will be the most significant development for disseminating the results of biomedical research in our lifetime. "

Sir Paul Nurse, Cancer Research UK

Your research papers will be:

- available free of charge to the entire biomedical community

- peer reviewed and published immediately upon acceptance

- cited in PubMed and archived on PubMed Central

- yours - you keep the copyright
BioMedcentral 